\title{
$\gamma$-Crystallin genes in carp: cloning and characterization
}

\author{
Tschining Chang ${ }^{1}$, Ching-Lung Lin ${ }^{2}$, Peng-Hui Chen ${ }^{2}$ and Wen-Chang Chang ${ }^{1.2}$ \\ 'Institute of Biological Chemistry, Academia Sinica, Taipei (Taiwan, R.O.C.) and ${ }^{2}$ Institute of Biochemical Sciences, National Taiwan \\ University, Taipei (Taiwan, R.O.C.)
}

(Received 6 August 1991)

Key words: $\gamma$-Crystallin; Gene structure; Amino acid sequence; (C. carpio)

The carp $\gamma$-crystallin gene family was found to be composed of at least three members: $\gamma \mathrm{m1}, \gamma \mathrm{m} 2$ and $\gamma \mathrm{m3}$. The encoded products are very similar to other known $\gamma$-crystallins but with their own peculiarities: (1) they all have a high methionine content: $12.4 \%, 14 \%$ and $8.4 \%$ in $\gamma \mathrm{ml}, \gamma \mathrm{m} 2$ and $\gamma \mathrm{m3}$, respectively; and (2) the amino acid sequences are aberrant in the region before connecting peptides and its corresponding region in motif 4 . Their protein structures might remain the same as those of other $\boldsymbol{\gamma}$-crystallins since they retain all the conserved amino acid residues essential for maintaining the loops in the protein structures.

The crystallins account for over $90 \%$ of the soluble proteins of the eye lens $[1,2]$. Immunologically, they can be distinguished into four major groups: $\alpha, \beta, \gamma$ and $\delta$ with $\delta$ restricted to birds and reptiles [3-5]. The short-range spatial order of crystallins was suggested to be important for lens transparency [6] and the $\gamma$-crystallins are of additional interest because of their implication in cataractogenesis [7]. Investigaters studying the lenses of human [8], rat [9] and mice [10] have concluded that the $\gamma$-crystallins are encoded by a multigene family. Those genes are highly conserved in structure with three exons and two introns in each gene. The $5^{\prime}$ exon is small and encodes three amino acids. The other two larger exons correspond to two structurally similar 'Greek key'-like domains of the protein. Recent studies on the promoter functions of these genes corfirmed that they are expressed in a tissue-specific manner [11,12].

In fish, $\gamma$-crystallins exhibit the conserved structural features of the known $\gamma$-crystallins but with their own peculiarities: high methionine content [13] and sequence aberrancy in motif 2 and its corresponding region in motif 4 of the protein (see text). Since the fish lives in a very different environment compared to

The sequence data in this paper have been submitted to the EMBL Data Library under the accession numbers X55945 (for $\gamma \mathrm{m1}$ ) and X55946 (for $\gamma \mathrm{m3}$ ).

Correspondence: W.C. Chang, Institute of Biological Chemistry, Academia Sinica, P.O. Box 23-106, Taipei, Taiwan, R.O.C. the terrestrial animals and exhibits remarkable species diversity [14], it is interesting to study lens crystallins of fish concerning the gene structure, regulation and molecular evolution. In this report we will present two nucleotide sequences and structural features of carp $\boldsymbol{\gamma}$-crystallin genes.

Five positive clones were obtained from a genomic library of $2 \cdot 10^{5}$ plaques with $\gamma \mathrm{m} 1 \mathrm{cDNA}$ as probe. After restriction enzyme mapping, four of them were found to be identical and designated as pEM64. The remaining positive clone was named pEM101. The complete nucleotide sequences are shown in Fig. 1. Both pEM64 and pEM101 contain a $\gamma$-crystallin gene with similar features: three exons and two introns on the basis of the known carp $\gamma$-crystallin cDNA sequences (Fig. 1). The first exon of both genes contains a 9 bp coding region with translation start codon. Exon 2 would encode motifs 1 and 2 of both gene products, while exon 3 corresponds to motifs 3 and 4 plus the $3^{\prime}$ untranslated region. All the introns are small. The first intron is 162 bp and 275 bp long for $\gamma \mathrm{m} 1$ and $\gamma \mathrm{m} 3$, respectively, while the second contains $138-139 \mathrm{bp}$, much smaller than those of mammalian $\gamma$-crystallins, most of which are larger than $1 \mathrm{~kb}$ in size $[8,16]$. All the introns start with GT and end with AG, in agreement with the GT/AG splice site rule. The $5^{\prime}$ and $3^{\prime}$ untranslated regions of both genes are short (Fig. 1). The polyadenylation signal, AATAAA, was found in the $3^{\prime}$ untranslated region of both genes. The termination codons, TAA (for both $\gamma \mathrm{m} 1$ and $\gamma \mathrm{m} 3$ ), were not far from the polyadenylation signals. 


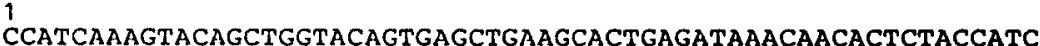

$r m 3$

$\gamma \mathrm{m} 2$

$\gamma_{\mathrm{m} 1} 1$

$Y \mathrm{~m} 3$

$\mathrm{Ym} 2$ CTGGACAGAATCCCACGAAGACTAGCAAAC

ATGGGCAAGg taatttgtttgaaataagtatgcttaatttttaagctttagtttcatat gtatgtatagggtggcettaatccagcagagtagagetgccaaaacagca $---$

tgcatcgagtttttatttaaagaa taaagacgaacagtatgcaattcagatctaaaaggc aatcctccataaattaataatgcaagatggctttttttttgtggaaatggtta

aattaattataaatttttgaaacact taaattttcaatcccatcaacagggtaattatattcaacatttgctacataacaagctctaatacaaatacctgttgtgtaa tgcaagccaaaaagccagttacttcaaatgttaaaggttactgtttacagagagcact

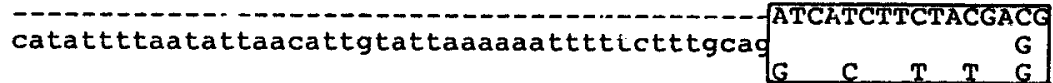
ACAGGAACTTCCAGGGCCGCAGCTATGACTGCATGAGCGACTGCTCTGATATCTCCTCTT

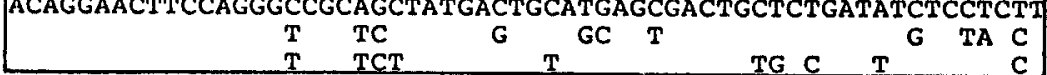
ACCTGAGCCGCGTTGGTTCAATCAGGGTGGAGAGTGGTTGTTTCATGGTCTATGAGCGCA

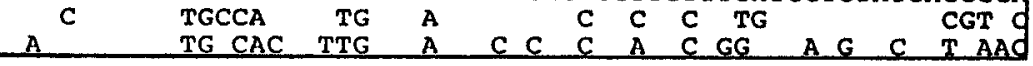
ACAGCTACATGGGGAACCAGTTCTTCCTGAGGAGGGGCGAGTACCATGATATGCAGCGCA

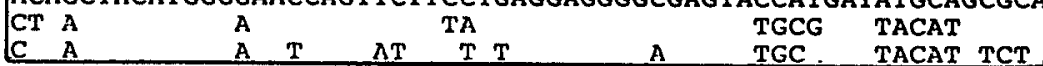
TGATGAGCATGGGCATGATTTTTGACACTATCAGATCCTGCCGCATGATTCCTCCAg tac

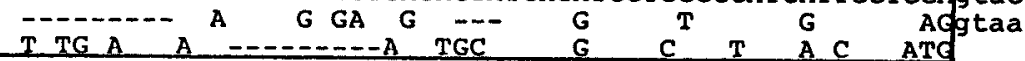
ggctctcactttgtttacagtattgtgtagatttagtaatgcacacctttaatgatatt caaaaataaacaataataatattatcatttaatcattaacaataatagttcaattge taaattgttcagactgtattgaattagaaatgcattgaatctgactgagtcaatacatt

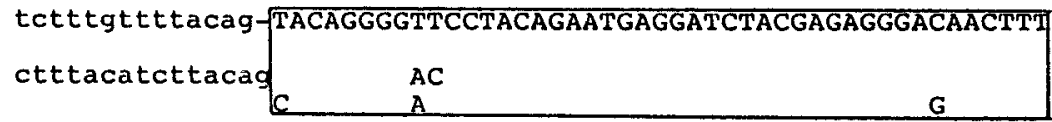
GGAGGACAGATGCACGAGGTGATGGATGACTGTGACAACATCATGGAACGTTACCGTATC

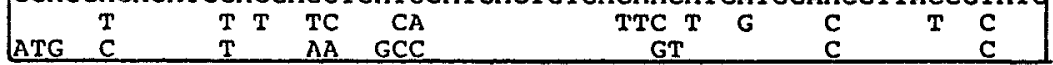
TCTGACTGGCAGTCTITTCATGTGATGGACGGCCACTGGCTCT TCTATGAGCAGCCACAQ

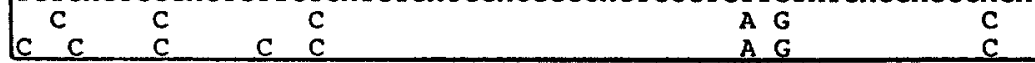
TACAGAGGCAGAAT'TTGTACTTCAGGCCTGGAGA TACAGGAGCTCAGAGATATGGGA G CGTA T A C A CA

GGA:-- G C A GCTCTG T AA A TG CTC GTAC AGTCYGAAACTATAAATGATAACATAATCATAAACAATAAATTTCTCACCATGCATTTTT 


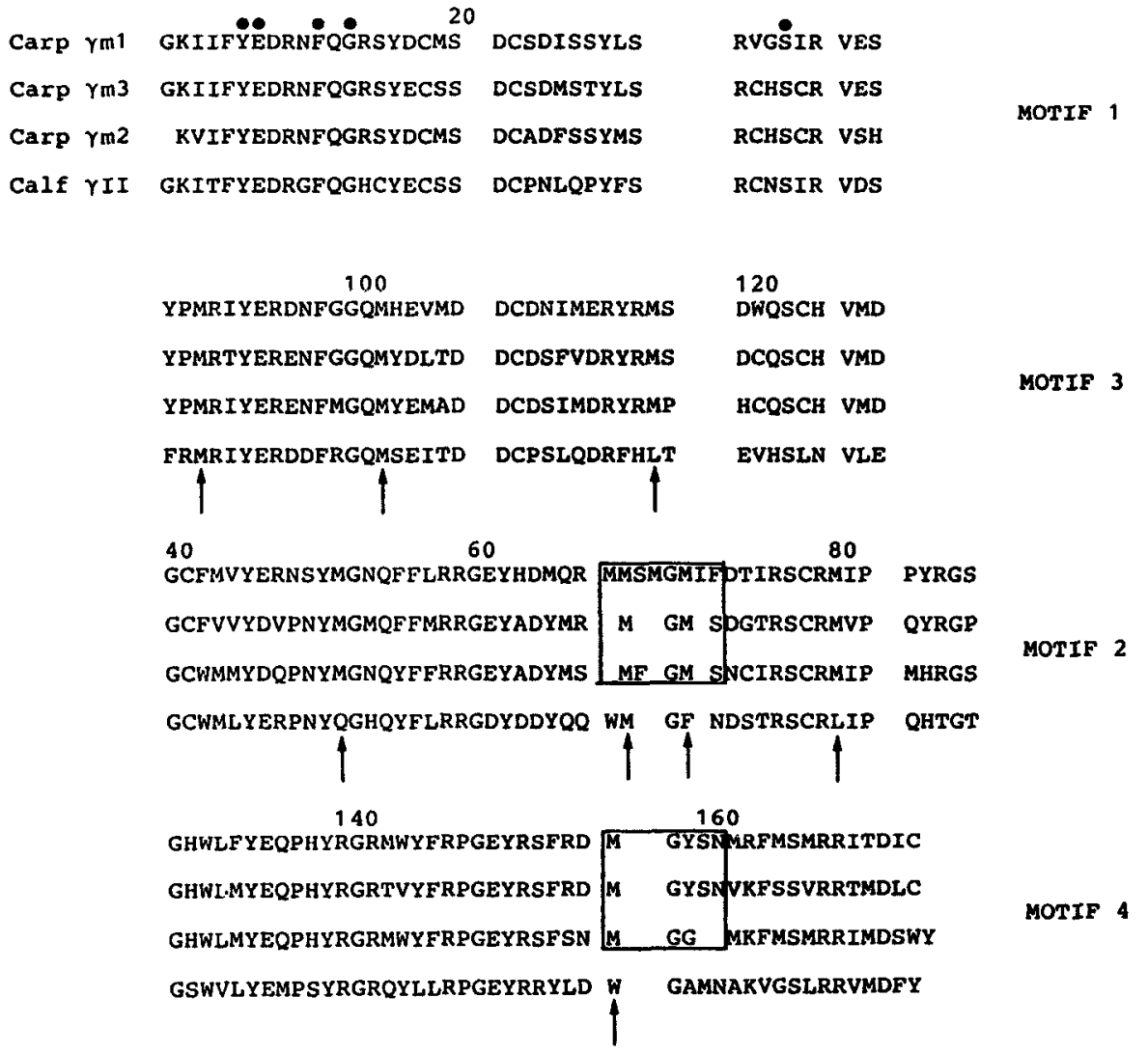

Fig. 2. Comparison of deduced amino acid sequences of $y \mathrm{~m} 1, \gamma \mathrm{m} 2$ and $\gamma \mathrm{m} 3$ with calf $\gamma$-II protein sequence. Conserved amino acids are labelled with filled circles. The sequences are so aligned that the topologically equivalent amino acid residues can be easily compared. The sequence-aberrant regions are boxed. The conserved methionine residues in all carp $\gamma$-crystallins are indicated by arrows. The numbering for calf $\gamma$-II sequence is used as reference.

The amino acid sequences derived from the exons of both genes show that they are closely related (Fig. 2). Comparison of pEM64 coding region with that of $\gamma \mathrm{m} 1$ cDNA [13] reveals only four base changes between them and five nucleotide differences in the $5^{\prime}$ and $3^{\prime}$ noncoding regions (data not shown). Therefore, pEM64 must contain the genomic equivalent of $\gamma \mathrm{ml}$ crystallin. Hereafter the symbol $\gamma \mathrm{m} 1$ will be used to denote this particular $\gamma$-crystallin and its genomic equivalents. The coding region of pEM101 is quite different from that of $\gamma \mathrm{m} 1$ and $\gamma \mathrm{m} 2$ [13] and it must encode a new species of carp $\gamma$-crystallin which will be symbolized as $\gamma \mathrm{m} 3$ to indicate that it is the third $\gamma$-crystallin with high methionine content ever found in carp. Compared with calf $\gamma$-II, the amino acid sequences of carp $\gamma$-crystallins are aberrant in the region from residues 68 to 72 before the connecting peptide and their counterpart in motif 4. In this region of motif $2, \gamma \mathrm{ml}$ has an insertion of four amino acids, $\gamma \mathrm{m} 2$ has a deletion of 2 amino acids in motif 4 and $\gamma \mathrm{m} 3$ has a deletion of one amino acid in motif 2 (Fig. 2). Therefore, these three proteins might have different fine structures. However, since those amino acids essential for loop-maintaining, i.e., Tyr-6, Glu-7, Gly-1 3, Ser-24 and their equivalents in

Fig. 1. Comparison of carp $\gamma$-crystallin genes. The carp (Cyprinus carpio) egg genomic library was constructed as described by Chiou et al. [15]. Positive clones were mapped, subcloned and reconfirmed by repeated hybridization with the $\gamma \mathrm{ml} \mathrm{cDNA}$ as probe. The nucleotide sequences were determined. Exons are shown by capital letters and introns in lower-case. The numbering of pEM64 ( $\gamma \mathrm{m} 1)$ is shown at right margin and begins with the transcription start site $(+1)$. Sequence of pEM101 $(\gamma \mathrm{m} 3)$ and $\gamma \mathrm{m} 2 \mathrm{cDNA}$ are aligned with respect to the coding regions which are boxed. Only the nucleotides different from that of pEM64 in the coding region are shown below it. Dashed lines are used to indicate deletions. The transcription start site in pEM1O1 is indicated by an asterisk. Polyadenylation signals are underlined. 


\section{TABLE I}

Percent homology of amino acid sequence between $\gamma m 1, \gamma m 2$ and $\gamma m 3$

\begin{tabular}{llll}
\hline & $\gamma \mathrm{m} 1 / \gamma \mathrm{m} 2$ & $\gamma \mathrm{ml} / \gamma \mathrm{m} 3$ & $\gamma \mathrm{m} 2 / \gamma \mathrm{m} 3$ \\
\hline Motif 1 & 72 & 82 & 74 \\
Motif 2 & 55 & 62 & 64 \\
Motif 3 & 78 & 76 & 78 \\
Motif 4 & 77 & 81 & 68 \\
\hline
\end{tabular}

the other motifs [17] are conserved, the protein skeleton should still be similar to those of mammalian $\gamma$-crystallins. The sequence comparison motif-by-motif between $\gamma \mathrm{m} 1, \gamma \mathrm{m} 2$ [13] and $\gamma \mathrm{m} 3$ reveals the lowest homology in motif 2 (Table I) instead of motif 3 which is the most diverse motif found in the mammalian $\gamma$-crystallin. The methionine content is high in $\gamma \mathrm{m} 3$,

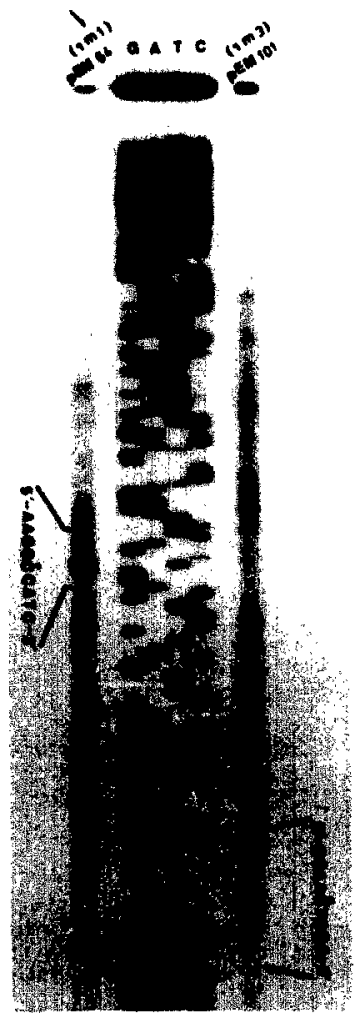

Fis. 3. The determination of the transcription start sites of $\gamma \mathrm{ml}$ and $\gamma \mathrm{m} 3$ by primer extension test. Two antisense primers complementary to a stretch or $17 \mathrm{bp}$ just upstream from the first start codon of both $\gamma \mathrm{ml}$ and $\gamma \mathrm{m} 3$ genomic sequences were used: S'-GATGGTAGAGTGTTGTT-3' for $\gamma \mathrm{ml}$ and 5'-GTTTGCTAGTCTTCGTG-3' for $\gamma \mathrm{m} 3$. Lanes $\mathrm{A}, \mathrm{G}, \mathrm{C}$ and $\mathrm{T}$ are sequence ladders. The nucleotide sequences denoted for lanes $\gamma \mathrm{ml}$ and $\gamma \mathrm{m3}$ are the sequences around the transcription start site which is indicated with an asterisk.
$8.4 \%$, although lower than that of $\gamma \mathrm{m} 1$ and $\gamma \mathrm{m} 2$. Most of the methionine residues of these three proteins are relatively conserved (Fig. 2). It is not clear whether these methionine residues are functionally important for carp $y$-crystallins. A detailed $X$-ray diffraction analysis might offer more insight into the possible roles in protein structure and the interactions between proteins and their environment.

In the 5' flanking regions, a TATA box was found for $\gamma \mathrm{ml}$, but two tandem repeats of TATA box were found for $\gamma \mathrm{m} 3$ (data not shown). The presence of such a 'double' TATA box in a promoter region is rare and its possible functional role should await further analysis. The cap site of the mRNA was determined by primer extension method as shown in Fig. 3 and was found to be a cytosine residue in both $\gamma \mathrm{m} 1$ and $\gamma \mathrm{m} 3$ genes. The primer extension experiments also indicate that both $\gamma \mathrm{ml}$ and $\gamma \mathrm{m} 3$ genes are expressed in the fish eye lens.

Although the gene structures of both $\gamma \mathrm{m} 1$ and $\gamma \mathrm{m} 3$ are very similar to that of known mammalian $\gamma$ crystallins, the existence of aberrent amino acid sequences and high methione content in the proteins may hint that the fish is one of the radiation points in the phylogenetic tree of $\gamma$-crystallin gene evolution. The exact size of this fish gene family and their detailed structural analyses will be needed to clarify this point.

\section{References}

1 Piatigorsky, J. (1984) Cell 38, 620-621.

2 Bloemendal, H. (1977) Science 197, 127-138.

3 McAvoy, J.W. (1978) J. Embryol. Exp. Morph. 45, 271-281.

4 Papaconstantinou, J. (1976) Science 156, 338-347.

5 McAvoy, J.W. (1978) J. Embryol. Exp. Morph. 44, 149-165.

6 Delaye, M. and Tardieu, A. (1983) Nature 302, 415-417.

7 Harding, J.J. (1981) Molecular and Cellular Biology of the Eye Lens, John Wiley and Sons, I New York.

8 Meakin, S.O., Breitman, M.L. and Tsui, L-C. (1985) Mol. Cell. Biol. 5, 1408-1414.

9 Den Dunnen, J.T., Moorman, R.J.M., Lubsen, N.H. and Schoenmakers, J.G.G. (1986) J. Mo. Biol. 189, 37-46.

10 Lok, S., Tsui, L-C., Shinohara, T., Piatigorsky, J., Gold, R., and Breitman, M.L. (1984) Nucleic Acids Res. 12, 4517-4529.

11 Lok, S., Breitman, M.L., Chepelinsky, A. B., Piatigorsky, J., Gild, RJ.M. and Tsui, L-C. (1985) Mol. Cell. Biol. 5, 2221-2230.

12 Wistow, G.J. and Piatigorsky, J. (1988) Annu. Rev. Biochem. 57, 479-504.

13 Chang, T., Jiang, Y-J., Chiou, S-H. and Chang, W-C. (1988) Biochim. Biophys. Acta 951, 226-229.

14 Powers, D.A. (1989) Science 246, 352-358.

15 Chiou, C.S., Chen, H.-T. and Chang, W-C. (1990) Biochim. Biophys. Acta 1087, 91-94.

16 Den Dunnen, J.T., Moorman, R.J.M., Lubsen, N.H. and Schoenmakers, J.G.G. (1986) J. Mol. Biol. 189, 37-46.

17 Wistow, G., Turnell, B., Summers, L., Slingsby, C., Moss, D., Miller, L., Lindley, P. and Blundell, T. (1983) J. Mol. Biol. 170, 175-202. 